\title{
EDITORIALS
}

\section{ORBITAL IMPLANTS: FOOD FOR THOUGHT}

Intraorbital implants that restore volume and improve prosthetic motility have been in use for over one hundred years. ${ }^{1}$ A wide variety of designs manufactured from metals, polymers or ceramics have been employed, often attesting as much to the ingenuity of the designer as to any real advantage of one implant over another. Complications seen with orbital implants include implant migration and extrusion, infection and poor motility of the overlying prosthesis. All too often attempts to minimise certain complications were associated with an increased incidence of other problems. For instance integrated implants, whereby the overlying prosthesis is coupled to the implant, offer enhanced prosthetic motility at the expense of increased rates of exposure or extrusion. Implant design was therefore, until recently, a compromise.

Bio-materials which become permanently integrated with, or incorporated into, host tissues have excited considerable interest in a variety of disciplines over the last ten to fifteen years. ${ }^{2-4}$ Integration is an important feature of so-called biocompatible materials, which thus represent a subgroup of extremely sophisticated and 'second generation' bio-materials. Coralline hydroxyapatite is one such material which, in spherical orbital implant form, has become a market leader in the United States over the last six years..$^{5-7}$ The coralline hydroxyapatite implant has a porous structure which permits fibrovascular ingrowth, or tissue integration, thereby minimising the risks of migration, extrusion and infection. A channel may be drilled into the hydroxyapatite implant when fibrovascular ingrowth is complete without risk of implant destabilisation; pegging of this channel facilitates direct coupling between the prosthesis and implant, thereby enhancing motility. The manufacture of spherical orbital implants incorporating bio-compatible and porous materials should, theoretically at least, minimise many of the complications which bedevilled earlier orbital implants. A number of newer materials with these characteristics are now manufactured in spherical orbital implant form, such as synthetic hydroxyapatite and porous polyethylene (Medpor). ${ }^{8}$ Unlike coralline hydroxyapatite (BioEye), drilling of these implants is not yet recommended by the manufacturers.

A number of important questions arise from these observations and these will be addressed in turn.

Are the theoretical advantages of the new generation porous implants borne out in clinical practice?

The majority of clinical data published to date relates to the coralline hydroxyapatite sphere. Undoubtedly progressive fibrovascular ingrowth occurs, as demonstrated by histopathological studies, technetiumlabelled bone scans and enhanced magnetic resonance imaging studies. As a consequence of this tissue integration, the rates of implant extrusion and/ or infection would appear to be lower than those described with more conventional implants, at least in the short to medium term. Volume augmentation equates with that achieved using the conventional spherical baseball implant. Prosthesis motility is reported as equal to, if not better than, that achieved with earlier implants without implant pegging. If the latter is undertaken this improves motility further, particularly with regard to fine saccadic or so-called conversational eye movements.

What are the differences between the currently available porous implants?

Coral basically comprises a carbonate exoskeleton surrounding interconnecting pores with diameters ranging from 140 to $160 \mu \mathrm{m}$. Coralline (or coral-like) hydroxyapatite is manufactured by first machining this carbonate exoskeleton into an appropriate shape. This acts as a template for a process (replamineformation) whereby the carbonate moieties are hydrothermally exchanged for phosphates, thus creating coralline hydroxyapatite. The manufacturers believe this to be the ideal porous implant because of the unique microstructural configuration so produced.

Synthetic hydroxyapatite and polyethylene are manufactured using a different process into appar- 
ently similar porous spherical forms, although evidence exists to suggest that porous interconnections in part may be sub-optimal. Whether or not this is of clinical significance remains unclear. Complete porous interconnections, and by inference complete fibrovascular ingrowth, may be clinically significant only when considering implant drilling (which is as yet recommended only for the coralline hydroxyapatite sphere).

A significant advantage of the porous polyethylene implant is that the extraocular muscles may be sutured directly to the implant surface. This is not possible with either the coralline or synthetic hydroxyapatite implant, which require a covering to which the muscles are sutured. The use of donor sclera is currently recommended, but in light of recent concern about potential slow virus transmission from donor sclera, alternative implant cover, in the form of autogenous fascia lata or synthetic meshes, has been described.

\section{Are porous implants associated with significant complications?}

The most significant problem relates to implant exposure. Exposure rates of up to $20 \%$ with coralline hydroxyapatite implants have been reported in the American literature, in comparison with a rate of 1$5 \%$ for silicone implants. ${ }^{9-11}$ An increased rate of exposure has also been reported with the porous polyethylene sphere. ${ }^{12}$ These results are mirrored by the experience of the Manchester group, ${ }^{13}$ who reported an exposure rate of $14 \%$ in twenty-eight primary implant patients. The latter group noted that chronic exposure did not appear to cause any problems in their group of patients and there were no cases of implant extrusion or migration. Nonetheless, further surgical intervention ranging from patch grafting to implant revision and replacement is usually necessary in the management of all but those cases with small and stable exposures. Implant drilling in certain cases has also led to implant exposure.

\section{What, if any, are the additional resource implications?}

Porous implants are more expensive than conventional implants. A synthetic polyethylene implant costs about five times as much as a silicone sphere; the coralline hydroxyapatite costs more than twenty times as much as a silicone implant. When put into the context of other implants, one coralline hydroxyapatite implant equates to three Charnley total hip replacement implants or fifteen intraocular lenses! Further additional costs occur if implant pegging is undertaken in the form of a second procedure, with additional imaging costs if the latter is felt appropriate.

These financial aspects should not be considered in isolation but more appropriately in the broader context of implant surgery. Additional in-patient and theatre expenditure arises when dealing with complications of implant surgery. If the use of porous implants results in less frequent complications, as suggested by a number of authors, then the additional initial costs of the implant will be rapidly offset under these circumstances. Adequate prosthetic support is an essential component of orbital implant surgery. The process of coralline hydroxyapatite implant pegging and subsequent prosthetics rehabilitation requires a dedicated service which for practical reasons is usually in-house. This facility is sadly lacking in all but a very small number of major units in the United Kingdom. The establishment of such services has significant additional resource implications.

Should the new generation porous implants be adopted routinely in ophthalmic clinical practice in the United Kingdom?

This is perhaps the most difficult question to answer. The majority opinion of specialist ophthalmic plastic surgeons in the United States suggests that this should be so. In the United Kingdom, however, bearing in mind the fundamental differences in the health care system on either side of the Atlantic, a number of ophthalmic plastic surgeons remain to be convinced.

The superior results obtained with the new generation implants may, to a greater or lesser extent, reflect the surgical expertise available in specialised units with a regular implant programme and attendant prosthetic support.

Long-term results in relation to porous implants are not yet known and a number of concerns (i.e. the material that is used to encase the implant; whether or not motility will be reduced in the long term consequent upon posterior implant fibrovascular ingrowth) remain unanswered. General ophthalmologists will, quite reasonably, look to the ophthalmic plastic fraternity for these answers. There is a pressing need for co-ordinated development and trials on a multi-centre basis; specialised and dedicated prosthetics support is an essential part of any implant programme. Until such time, the ad hoc adoption of aggressively marketed novel implants should be tempered with caution. Without doubt, the introduction of bio-materials technology tailored to clinical practice is an exciting development, but in an increasingly financially aware environment we must be sure that the benefits to the patient outweigh the costs involved.

RICHARD Downes

\section{References}

1. Mules PH. Evisceration of the globe, with artificial vitreous. Trans Ophthalmol Soc UK 1885;5:200-6. 
2. White EW, Weber JN, Roy DM, Owen EL. Replamineform porous biomaterials for hard tissue implant applications. J Biomed Mat Res Symp 1975;6:23-7.

3. Tanner KE, Downes RN, Bonfield W. Clinical applications of hydroxyapatite reinforced materials. Br Ceram Trans 1994;93:104-6.

4. Jonck LM, Grobbelaar CJ. A glass ionomer for reconstructive surgery. Ionogran: an ionomeric micro implant. A biological evaluation. J Clin Mat 1992;9:85-103.

5. Perry AC. Integrated orbital implants. Adv Ophthalmic Plast Reconstr Surg 1988;8:75-81.

6. Shields CL, Shields JA, Eagle RC Jr, De Potter P. Histopathologic evidence of fibrovascular ingrowth four weeks after placement of the hydroxyapatite orbital implant. Am J Ophthalmol 1991;111:363-6.

7. Shields CL, Shields JA, De Potter P. Hydroxyapatite orbital implant after enucleation: experience with initial 100 consecutive cases. Arch Ophthalmol 1992;110:333-8.

8. Rubin PAD, Popham JK, Bilyk JR, Shore JW. Comparison of fibrovascular ingrowth into hydroxya- patite and porous polyethylene orbital implants. Ophthalmic Plast Reconstr Surg 1994;10:96-103.

9. Buettner H, Bartley GB. Tissue breakdown and exposure associated with orbital hydroxyapatite implants. Am J Ophthalmol 1992;113:669-73.

10. Goldberg RA, Holds JB, Ebrahimpour J. Exposed hydroxyapatite orbital implants: report of six cases. Ophthalmology 1992;99:831-6.

11. Shields CL, Shields JA, De Potter P, Singh AD. Problems with the hydroxyapatite orbital implant: experience with 250 consecutive cases. $\mathrm{Br} \mathrm{J}$ Ophthalmol 1994;78:702-6.

12. Heidi D, Remulla MD, Peter AD, Rubin MD, John W, Shore MD, Francis C, Sutula MD, Daniel J, Townsend MD, John J, Woog MD, Kurt V, Jahrling BCO. Complications of porous spherical orbital implants. Ophthalmology 1995;102:586-93.

13. Ashworth JL, Rhatigan M, Sampath R, Brammar R, Sunderland S, Leatherbarrow B. The hydroxyapatite orbital implant: a prospective study. Eye 1996; 10:29-37.

\section{ACUTE SYMPTOMATIC TOXOPLASMA RETINOCHOROIDITIS}

The clinical diagnosis of acute toxoplasma retinochoroiditis is made on the characteristic features of intraocular inflammation with a focal retinitis adjacent to an old chorioretinal scar. There has been heightened professional and public awareness of congenital toxoplasmosis recently, particularly with respect to the value of screening (and treating) antenatal disease. ${ }^{1}$ However, it is apparent that little is known about the impact of this infection on longterm visual morbidity, less on the most appropriate methods of laboratory diagnosis and management and practically nothing on the way in which the parasite enters and encysts in retinal tissue or how it recurs many years later to produce symptomatic disease.

Epidemiological data derived from clinic-based follow-up studies have suggested that retinochoroidal lesions occur in over $80 \%$ of people with congenital toxoplasmosis. ${ }^{2,3}$ The proportion of affected people who eventually develop symptoms is not known. A recent study has, however, shown that the incidence of symptomatic disease in Britain might be much lower than that expected from the predicted prevalence of retinochoroidal lesions. ${ }^{4}$ This study showed an incidence of symptomatic disease in the native-born population of $0.4 / 100000$ per year. Therefore, assuming that each patient suffers a mean of two symptomatic attacks per lifetime, 100 people born in Britain may be affected each year about one-fifth of the estimated 500-600 people born with congenital disease. ${ }^{1}$ Interestingly, toxoplasma retinochoroiditis was almost 100 times commoner in patients born in West Africa. Since this was not a follow-up study the long-term impact on visual morbidity was not specifically addressed, although it was noted that vision transiently fell to $6 / 12$ or less in two-thirds of patients. The findings suggested that prenatal screening for toxoplasmosis in Britain to prevent visual loss might be of limited benefit.

It is becoming increasingly recognised that some acute symptomatic toxoplasma retinochoroiditis may be the result of acquired rather than congenital infection. In the absence of clinical or serological indicators of recent infection such cases are indistinguishable from congenital disease. Toxoplasma retinochoroiditis has been reported in siblings in Brazil and $20 \%$ of adults in this population had toxoplasma chorioretinal scars despite an incidence of a positive IgM against the organism in cord blood of $1 \% .^{5}$ Acquired disease is also reported to occur in immunocompromised patients but is much less common than toxoplasma encephalitis in this group. ${ }^{6}$ Confirmation of the diagnosis in all patients by laboratory testing is difficult given the high seroprevalence of antibodies to the parasite in most populations. Whilst IgM positivity has been taken to imply a recently acquired infection, current techniques are so sensitive that a positive result may last for 18 months after initial infection and IgM positivity has also been reported in recurrent disease. ${ }^{7}$ Newer techniques which involve the detection of parasite DNA in ocular fluid by the polymerase chain reaction are promising, but require further refinement.

There is no one drug regime that will eliminate 